\title{
PELVIC OSTEOSARCOMA AFTER RADIATION THERAPY OF UTERINE CERVICAL CANCER: A CASE REPORT
}

Dias, A.L.R.; Sartorelli, V.; Monte, A.A.; Ferreira, A.F.A.; Costa, R.L.R.; Forghieri, M.C.G. Instituto Brasileiro de Controle do Câncer - Departament of Ginecology, São Paulo, Brazil

\section{Objectives:}

The objective is to report a rare case of a patient with pelvic osteosarcoma after radiation therapy of uterine cervical cancer.

\section{Methods:}

The necessary data was obtained by medical chart review, interview with the patient, image diagnose exams and literature review.

\section{Introduction:}

The development of osteosarcoma at the same time of another cancer is a rare fact. The risk factors and the origin of this tumor remains controversial. It is clearly that ionizing radiation can induce sarcoma. It is dificult to make systematic studies due to the rarity of these cases.

\section{Case report:}

A 58-year-old woman who received pelvic irradiation for stage IB2 uterine cervical cancer 7 years before was diagnosed with postradiation osteosarcoma of the iliac right bone.

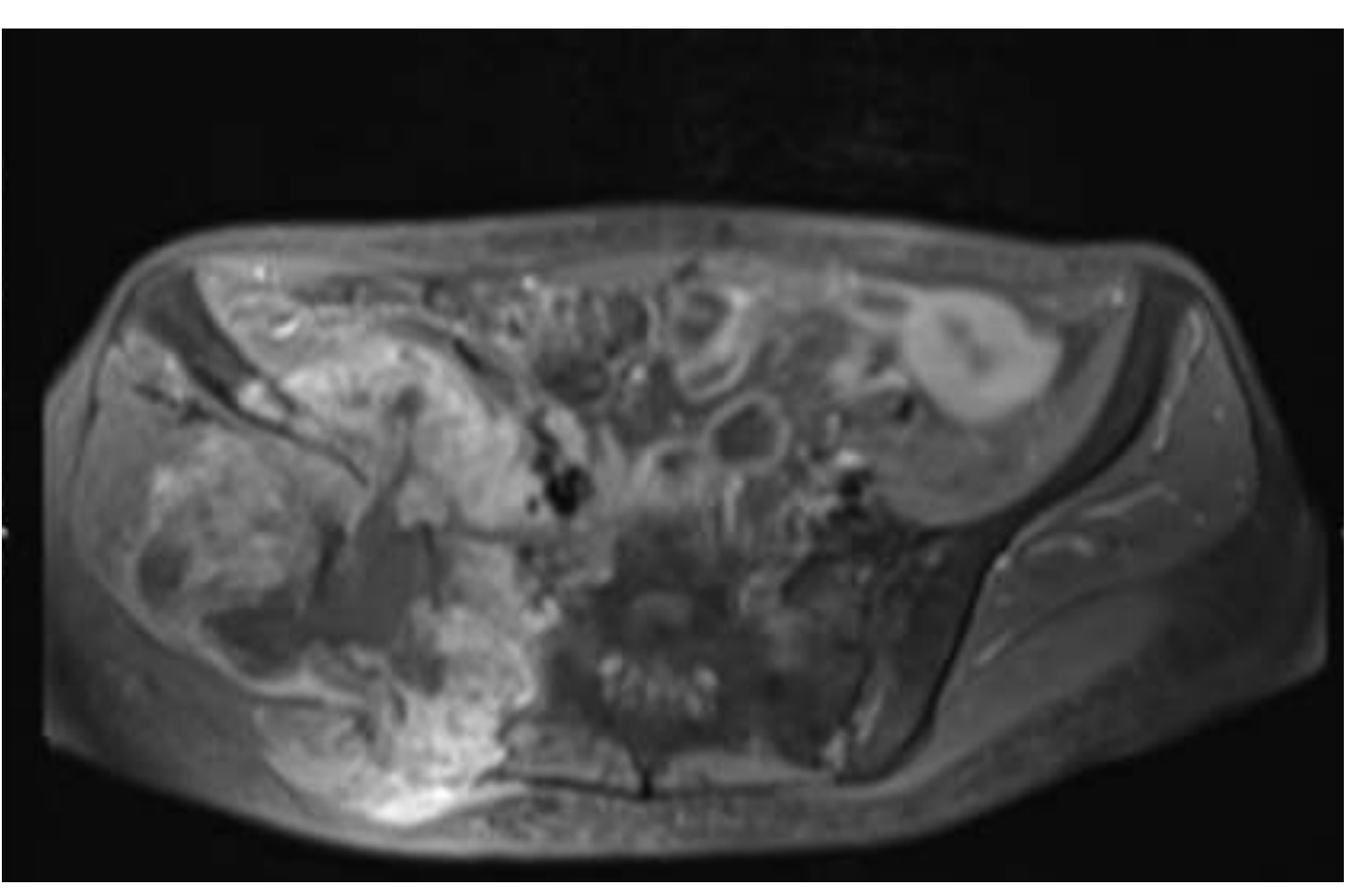

Figure 1: Osteosarcoma on the iliac right bone

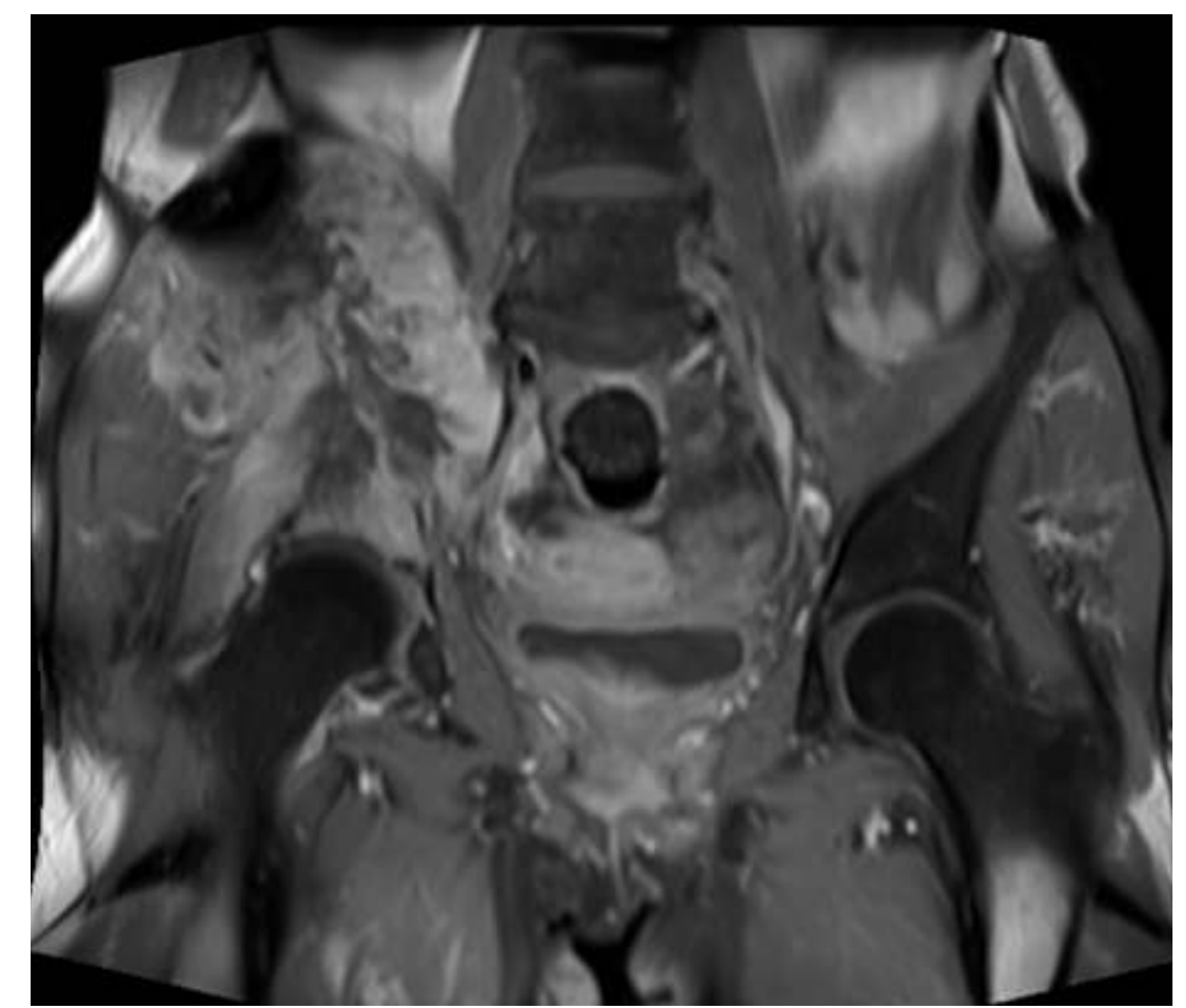

Figure 2: Osteosarcoma on the iliac right bone

\section{Discussion:}

For uterine cervical cancer stages IB2 to IVA radiotherapy associated with cisplatin for up to six cycles (chemoradiotherapy) has been the first line treatment choice with good results.

The sarcomas post radiotherapy are rare. Usually appear 10 to 14.3 years posttreatment, the incidence comprises about $0.1 \%$ of all cancer cases and women are more affected because gynecological cancers are more frequently subjected to radiotherapy with a long-term survival.

\section{Conclusion:}

This case is relevant due to the lenght of time over wich patients treated with radiotherapy may remain to be diagnosed with bone disease. A post-radiation sarcoma should be considered and differentiated from bone metastasis. To early diagnose is important to allow full treatment, providing a longer disease free survival. 